\title{
SOME APPLICATIONS OF GELFAND PAIRS TO NUMBER THEORY
}

\author{
BENEDICT H. GROSS
}

The classical theory of Gelfand pairs has found a wide range of applications, ranging from harmonic analysis on Riemannian symmetric spaces to coding theory. Here we discuss a generalization of this theory, due to Gelfand-Kazhdan, and Bernstein, which was developed to study the representation theory of $p$-adic groups. We also present some recent number-theoretic results, on local $\epsilon$-factors and on the central critical values of automorphic $L$-functions, which fit nicely into this framework.

\section{CoMpact PAIRS}

Let $G$ be a compact topological group. By a representation of $G$ we will mean a continuous homomorphism from $G$ to the group of unitary operators on a Hilbert space $V$. If $V$ and $W$ are two representations of $G$, the complex vector space $\operatorname{Hom}_{G}(V, W)$ consists of all continuous linear transformations from $V$ to $W$ which commute with the action of $G$.

We say $V$ is an irreducible representation of $G$ if and only if there are no nontrivial closed subspaces of $V$ which are $G$ invariant. The irreducible representations of $G$ are all finite dimensional [D, Chapter 3]. Let $V$ be a fixed irreducible representation. Then $V$ has, up to scaling, a unique $G$-invariant Hermitian structure, and any linear map from $V$ to a Hilbert space $W$ is continuous. If $W$ is a representation of $G$, we define the multiplicity of $V$ in $W$ as the dimension of the complex vector space $\operatorname{Hom}_{G}(V, W)$. We will only consider those representations $W$ (often called admissible) such that $d_{i}=\operatorname{dim}_{\operatorname{Hom}_{G}}\left(V_{i}, W\right)$ is finite, for all irreducible representations $V_{i}$ of $G$. In this case, $W$ decomposes as a Hilbert space direct sum: $W \simeq \hat{\bigoplus}_{i} d_{i} V_{i}$. We say $W$ is multiplicity-free if $d_{i} \leq 1$ for all $i$.

Received by the editors July 5, 1990 and, in revised form, September 13, 1990. 1980 Mathematics Subject Classification (1985 Revision). Primary 20C15, $11 \mathrm{~F} 66,11 \mathrm{~F} 67$. 
Lemma 1.1. $W$ is multiplicity-free if and only if $\operatorname{Hom}_{G}(W, W)=$ $\operatorname{End}_{G}(W)$ is a commutative algebra.

Proof. $\operatorname{End}_{G}(W)$ contains the direct sum of matrix rings

$$
\operatorname{End}_{G}\left(d_{i} V_{i}\right)=\operatorname{Mat}\left(d_{i}, \mathbf{C}\right)
$$

and is contained in their direct product, by Schur's lemma. Hence it is commutative iff all $d_{i} \leq 1$.

Let $H$ be a closed subgroup of $G$, so $H$ is also compact. We say that $(G, H)$ is a Gelfand pair, or that $H$ is a Gelfand subgroup of $G$, if the following three equivalent conditions hold.

(1.2) For every irreducible representation $V$ of $G$ the space $\operatorname{Hom}_{H}(V, \mathbf{C})$ of $H$-invariant linear forms has dimension $\leq 1$.

(1.3) The representation of $G$ by right translations on $W=L^{2}(H \backslash G)$ is multiplicity free.

(1.4) The sub-algebra $L^{1}(H \backslash G / H)$ of bi- $H$-invariant functions in $L^{1}(G)$ is commutative under convolution.

In (1.3) and (1.4) the spaces $L^{2}$ and $L^{1}$ are defined using the unique Haar measure of volume 1 on $G$, and the associated $G$ invariant measure on $H \backslash G$. The equivalence of (1.2) and (1.3) follows from Frobenius reciprocity, which gives an isomorphism

$$
\operatorname{Hom}_{G}\left(V, L^{2}(H \backslash G)\right) \Rightarrow \operatorname{Hom}_{H}(V, \mathbf{C}) \text {. }
$$

The equivalence of (1.3) and (1.4) follows from the injection

$$
L^{1}(H \backslash G / H) \hookrightarrow \operatorname{End}_{G}\left(L^{2}(H \backslash G)\right),
$$

where $L^{1}$ acts on $L^{2}$ by convolution. Since the image contains the $G$-endomorphisms of finite rank, the commutativity of

$$
L^{1}(H \backslash G / H)
$$

forces the commutativity of $\operatorname{End}_{G}\left(L^{2}(H \backslash G)\right)$.

Since $V$ is finite dimensional, and gives a semisimple representation of $H$, condition (1.2) is equivalent to

(1.5) For every irreducible representation $V$ of $G$, the space $V^{H}$ of $H$-invariant vectors has dimension $\leq 1$. 
which is the usual definition of Gelfand pairs. Indeed, $\operatorname{Hom}_{H}(V, \mathbf{C})$ is canonically identified with the dual space $\left(V^{H}\right)^{*}$, as any $H$ invariant linear form vanishes identically on the orthogonal complement of $V^{H}$ in $V$.

\section{GeLFAND'S LEMMA}

If one knows the irreducible representations of $G$ explicitly, condition (1.5) is probably the easiest to check. But condition (1.4) is extremely attractive, as it only makes reference to the internal structure of the pair $(G, H)$. Recall that an anti-involution $i$ of $G$ is a bijection $i: G \rightarrow G$ which satisfies $i(g h)=i(h) i(g)$ and $i^{2}=1$; a typical anti-involution is inversion $i(g)=g^{-1}$.

Lemma 2.1 (Gelfand [Ge]). Assume that $i$ is an anti-involution of $G$, which stabilizes $H$ and acts trivially on the double coset space $H \backslash G / H$. Then the algebra $L^{1}(H \backslash G / H)$ is commutative and $(G, H)$ is a Gelfand pair.

We omit the proof, which can be found in [L, p. 53]. Here is a typical application [ $\mathrm{Di}, \mathrm{p}$. 59]. Assume $G$ acts two-point transitively on a metric space $X$. By this we mean that if $(x, y)$ and $\left(x^{\prime}, y^{\prime}\right)$ are pairs of points in $X$ with $d(x, y)=d\left(x^{\prime}, y^{\prime}\right)$, then there is an element $g \in G$ such that $g x=x^{\prime}$ and $g y=y^{\prime}$. Let $H$ be the stabilizer of the point $x_{0}$. Then $G / H=X$ and the class of $g$ in $H \backslash G / H$ depends only on the distance $d\left(x_{0}, g x_{0}\right)$. Since $d(x, g x)=d\left(g^{-1} x, x\right)=d\left(x, g^{-1} x\right)$, we see that $i(g)=$ $g^{-1}$ is an anti-involution which acts trivially on $H \backslash G / H$. Hence, by Gelfand's lemma, we have shown the following.

Proposition 2.2. If $G$ acts 2-point transitively on a metric space $X$ and $H$ is the stabilizer of a point, then $(G, H)$ is a Gelfand pair.

Another application of Gelfand's lemma is

Proposition 2.3. Assume that $\sigma$ is an involution of $G$ such that every element $g \in G$ can be written in the form $g=h \cdot z$ with $\sigma(h)=h$ and $\sigma(z)=z^{-1}$. Then the subgroup $H$ fixed by $\sigma$ is a Gelfand subgroup of $G$.

Proof. Let $i$ be the anti-involution $i(g)=\sigma\left(g^{-1}\right)$; then $i$ acts trivially on $H \backslash G / H$.

To make new pairs from old, the following result is useful.

Lemma 2.4. (a) If $\left(G_{1}, H_{1}\right)$ and $\left(G_{2}, H_{2}\right)$ are Gelfand pairs, so is $\left(G=G_{1} \times G_{2}, H=H_{1} \times H_{2}\right)$. 
(b) If $(G, H)$ is a Gelfand pair and $H \rightarrow H^{\prime}$ is a continuous group homomorphism, then the subgroup $H$ diagonally embedded in $H^{\prime} \times G$ is Gelfand if and only if: for every pair of irreducible representations $W$ of $H^{\prime}$ and $V$ of $G$ we have $\operatorname{dim} \operatorname{Hom}_{H}(W, V) \leq 1$.

(c) The subgroup $H$ diagonally embedded in $H \times G$ is Gelfand if and only if the restriction of every irreducible representation $V$ of $G$ to $H$ is multiplicity free.

Proof. (a) This follows from (1.5). The irreducible representations $V$ of $G$ all have the form $V_{1} \otimes V_{2}$, where $V_{1}$ is an irreducible representation of $G_{1}$ and $V_{2}$ is an irreducible representation of $G_{2}$. We have $\operatorname{dim} V^{H}=\operatorname{dim} V_{1}^{H_{1}} \cdot \operatorname{dim} V_{2}^{H_{2}}$.

(b) The irreducible representations of $H^{\prime} \times G$ have the form $W \otimes V$. But $(W \otimes V)^{H}=\operatorname{Hom}_{H}\left(W^{*}, V\right)$.

(c) This follows from (b), taking $W$ an arbitrary irreducible representation of $H=H^{\prime}$.

Proposition 2.5 (cf. [M]). Let $H$ be a compact group. Then $H$ is a Gelfand subgroup (diagonally embedded) of $G=H \times H$. Furthermore, $H$ is a Gelfand subgroup of $H \times H \times H$ if and only if the tensor product $U_{1} \otimes U_{2}$ of any two irreducible representations of $\mathrm{H}$ is multiplicity-free.

Proof. The first claim follows trivially from part (c) of Lemma 2.4. The second also follows from this lemma, as the restriction of the representation $U_{1} \otimes U_{2}$ of $G$ to $H$ is precisely the tensor product $U_{1} \otimes U_{2}$.

Note 2.6. One can also show that $H$ is a Gelfand subgroup of $G=$ $H \times H$ using Lemma 2.1. Indeed, the anti-involution $i\left(h_{1}, h_{2}\right)=$ $\left(h_{2}^{-1}, h_{1}^{-1}\right)$ of $G$ acts trivially on the double coset space $H \backslash G / H$. We remark that this double coset space may be identified with the set of conjugacy classes in $H$, under the map taking the double class of $g=\left(h_{1}, h_{2}\right)$ to the conjugacy class of $h_{1} h_{2}^{-1}$.

\section{SOME COMPACT EXAMPLES}

We use the results of the previous section to give some concrete examples. Let $X$ be the $n$-sphere in $\mathbf{R}^{n+1}$, with its standard geometry. The special orthogonal group $G=S O_{n+1}$ acts 2-point transitively on $X$ with stabilizer $H=S O_{n}$, and the double coset space $H \backslash G / H$ is identified with the interval of distances $[0, \pi]$ from $g x_{0}$ to $x_{0}$. Hence, by Proposition 2.2, $\left(S O_{n+1}, S O_{n}\right)$ is a Gelfand pair. 
In fact, the restriction of any irreducible representation of $S O_{n+1}$ to $S O_{n}$ is multiplicity-free (cf. [D, Chapter 2] for the case $n=2)$, so $\left(S O_{n+1} \times S O_{n}, S O_{n}\right)$ is a Gelfand pair by Lemma 2.4, part (c). We note that $S O_{3}=\mathbf{H}^{*} / \mathbf{R}^{*}$, where $\mathbf{H}$ is the algebra of Hamilton's quaternions, and $S O_{2}=\mathbf{C}^{*} / \mathbf{R}^{*}$. This is a special case $(F=\mathbf{R})$ of

Proposition 3.1. Let $F$ be a local field, with $F \neq C$. Let $D$ be a quaternion division algebra with center $F$, and let $K$ be a separable quadratic field extension of $F$. Then $K$ embeds as a subfield of $D$, and $H=K^{*} / F^{*}$ is a closed subgroup of the compact group $G=D^{*} / F^{*}$.

The group $H$ is a Gelfand subgroup of $H \times G$, and the group $G$ is a Gelfand subgroup of $G \times G \times G$.

Proof. The first paragraph follows from the arithmetic of local quaternion algebras [V, Chapter II], which also shows that the normalizer of $H$ in $G$ is generated by a nontrivial involution $\alpha \in G-H$.

To show $(H \times G, H)$ is Gelfand, we use the anti-involution $i(h, g)=\left(h, \alpha g^{-1} \alpha^{-1}\right)$. To show $(G \times G \times G, G)$ is Gelfand, we use the anti-involution $i\left(g_{1}, g_{2}, g_{3}\right)=\left(g_{1}^{-1}, g_{2}^{-1}, g_{3}^{-1}\right)$. We leave the details of checking that these anti-involutions act trivially on the respective double coset spaces to the reader.

Note 3.2. When $F=\mathbf{R}$ the above proposition shows that the tensor product of any two irreducible representations of $\mathrm{G}=\mathrm{SO}_{3}$ is multiplicity-free. This is also true for its universal covering group $\widetilde{G}=S U_{2}$, by the Clebsch-Gordan formula [D, p. 13].

If $G$ is a compact, connected, semisimple Lie group with involution $\sigma$, the fixed subgroup $H$ is always a Gelfand subgroup of $G$. This is proved using the action of $\sigma$ on the Lie algebra $\mathfrak{g}$ and Proposition 2.3; the involutions which can occur were classified by Cartan (cf. [H, Chapter X, §3]). These Gelfand pairs $(G, H)$ are called symmetric: they include $\left(S O_{n+1}, S O_{n}\right)$ - take $\sigma$ to be conjugation by the reflection $\left(\begin{array}{cc}+1_{n} & \\ & -1\end{array}\right)$ in $O_{n+1}$.

When $G$ is a compact, connected, semisimple Lie group, let $G_{\mathrm{C}}$ be the complexified group, which is a semisimple algebraic group over $\mathbf{C}$. Let $B_{\mathrm{C}}$ be a Borel subgroup of $G_{\mathrm{C}}$ and $X(\mathbf{C})=$ $G_{\mathrm{C}} / B_{\mathrm{C}}$ the associated (projective) flag variety of $G_{\mathrm{C}}$. Let $H$ be a reductive, closed subgroup of $G$ with complexification $H_{\mathrm{C}}$. 
Then $(G, H)$ is a Gelfand pair if and only if $H_{\mathrm{C}}$ has an open orbit on $X(\mathbf{C})$ [K-V]. The pairs $(G, H)$ with $G$ simple have been determined by Krämer [K], who also determines which irreducible representations $V$ of $G$ have an $H$-fixed vector. These include the familiar symmetric pairs, but also some others, such as $\left(S O_{2 n+1}, U_{n}\right)$.

Some interesting Gelfand pairs of finite groups correspond to miniscule representations $M$ of simple Lie algebras $\mathfrak{g}$ over $\mathbf{C}$ [Bo, $\S 13]$. Let $G$ be the Weyl group of $\mathfrak{g}$; then $G$ permutes the weights in $M$ 2-point transitively, given the metric $d(x, y)=|x-y|$ induced from the Euclidean structure on the weight lattice. If $H$ is the stabilizer of a weight, then $(G, H)$ is a Gelfand pair. Taking $\mathfrak{g}=s \ell_{n}$ and $M=\Lambda^{k} \mathbf{C}^{n}$ gives the pair $\left(S_{n}, S_{k} \times S_{n-k}\right)$ where $S_{n}$ is the symmetric group on $n$ letters. Taking $\mathfrak{g}=s O_{2 n+1}$ and $M$ the spinorial representation of dimension $2^{n}$ gives the pair $\left(S_{n} \times(\mathbf{Z} / 2)^{n}, S_{n}\right)$. Taking $\mathfrak{g}=s p_{2 n}$ and $M$ the standard representation of dimension $2 n$ gives the pair $\left(S_{n} \ltimes(Z / 2)^{n}, S_{n-1} \ltimes\right.$ $\left.(\mathbf{Z} / 2)^{n-1}\right)$.

For more examples of specific compact Gelfand pairs, see [Di, p. 59ff.] and the references cited therein.

\section{TOTALLY DISCONNECTED GROUPS}

Many authors have considered the generalization of Gelfand pairs to locally compact groups $G$, but the closed subgroup $H$ is usually assumed to be compact [D, Chapter 8]. This hypothesis is too restrictive for various applications in number theory. We will consider the case when $G$ is locally compact and totally disconnected, following Gelfand-Kazhdan [Ge-K] and Bernstein [B]. This gives a reasonable generalization of Gelfand pairs, assuming only that $H$ is closed and that $H \backslash G$ carries a $G$-invariant measure.

By a (smooth) representation of a locally compact, totally disconnected group $G$ we mean a homomorphism from $G$ to the group of invertible linear transformations of a complex vector space $V$, such that every vector $v \in V$ has an open stabilizer in $G$. We only consider representations which are admissible: by this we mean that the subspace $V^{K}$ fixed by any compact open subgroup $K$ of $G$ has finite dimension. As usual, we say $V$ is irreducible if there are no nontrivial $G$-invariant subspaces in $V$. If $V$ and $W$ are two representations of $G$, we let $\operatorname{Hom}_{G}(V, W)$ 
denote the complex vector space of all linear transformations from $V$ to $W$ which commute with the action of $G$.

Let $V$ be a representation of $G$ and let $V^{*}=\operatorname{Hom}(V, \mathbf{C})$ be the dual vector space. There is a canonical bi-linear form $\langle$,$\rangle :$ $V \times V^{*} \longrightarrow \mathbf{C}$ defined by $\langle v, \ell\rangle=\ell(v)$ and we define an action of $G$ on $V^{*}$ by the formula $\langle g v, g \ell\rangle=\langle v, \ell\rangle$. The contragredient representation $V^{\prime} \subseteq V^{*}$ of $G$ is on the subspace of linear forms $\ell$ which are fixed by some open subgroup $K_{\ell}$ in $G$. This is a (smooth and admissible) representation of $G$, and the restriction of $\langle$,$\rangle gives a nondegenerate pairing V \times V^{\prime} \longrightarrow \mathrm{C}$ which is $G$-invariant. The representation $V^{\prime}$ is irreducible if and only if $V$ is, and the canonical map $V \rightarrow V^{\prime \prime}$ is an isomorphism. For a sketch of the proof of these claims, see [C, 1.5].

Let $H$ be a closed subgroup of $G$ such that $H \backslash G$ carries a $G$-invariant measure. We say that $(G, H)$ is a Gelfand pair if the following condition is satisfied

For every irreducible representation $V$ of $G$ we have $\operatorname{dim} \operatorname{Hom}_{H}(V, \mathbf{C}) \cdot \operatorname{dim} \operatorname{Hom}_{H}\left(V^{\prime}, \mathbf{C}\right) \leq 1$.

In all the cases we will consider,

$$
\operatorname{dim} \operatorname{Hom}_{H}(V, \mathbf{C})=\operatorname{dim} \operatorname{Hom}_{H}\left(V^{\prime}, \mathbf{C}\right),
$$

so condition (4.1) reduces to the usual condition (1.2). However, I have not been able to establish the equality in the general case, and condition (4.1) is the one that arises most naturally. It can be restated as
For every irreducible representation $V$ of $G$ the space $\operatorname{Hom}_{H \times H}\left(V \otimes V^{\prime}, \mathbf{C}\right)$ of $H \times H$-invariant linear forms has dimension $\leq 1$.

Indeed, the definition (4.1) was motivated by the following generalization of Gelfand's lemma, due to Gelfand and Kazhdan [Ge-K]. Recall that the space $S(G)^{*}$ of distributions on $G$ is the linear dual of the vector space $S(G)$ of locally constant functions with compact support. The group $G \times G$ acts on $S(G)^{*}$, via right and left translation of functions in $S(G)$.

Proposition 4.2. Assume that $i$ is an anti-involution of $G$, which stabilizes $H$ and acts trivially on the subspace of $H$-bi-invariant distributions in $S(G)^{*}$. Then $(G, H)$ is a Gelfand pair.

Proof (cf. [P, Lemma 4.2]). Let $V$ be an irreducible representation of $G$ and let $\ell: V \rightarrow \mathrm{C}$ and $m: V^{\prime} \rightarrow \mathrm{C}$ be nonzero, $H$-invariant 
linear forms. These give surjective $G$-linear maps $F_{\ell}: S(G) \rightarrow V^{\prime}$ and $F_{m}: S(G) \rightarrow V^{\prime \prime}=V$, defined by

$$
\begin{gathered}
F_{\ell}(f)(v)=\int_{G} f(g) \ell(g v) d g \\
F_{m}(f)\left(v^{\prime}\right)=\int_{G} f(g) m\left(g v^{\prime}\right) d g
\end{gathered}
$$

for $f \in S(G)$. Since $V^{\prime}$ and $V$ are irreducible, the linear map $F_{\ell}$ is determined, up to scalars, by its kernel, and similarly $F_{m}$ is determined, up to scalars, by its kernel.

We compose with the $G$-invariant pairing $\langle\rangle:, V \times V^{\prime} \rightarrow \mathbf{C}$ to obtain a linear map

$$
B: S(G) \otimes S(G) \underset{F_{m} \otimes F_{\ell}}{\longrightarrow} V \otimes V^{\prime} \underset{\langle,\rangle}{\longrightarrow} \text {. }
$$

Thus $B$ may be viewed as a distribution on $G \times G$ which is right invariant under $H \times H$ and left invariant under $G$ (embedded diagonally). We have the formula: $B\left(f_{1}, f_{2}\right)=m\left(F_{\ell}\left(f_{1} * f_{2}\right)\right)$, where $f_{1} * f_{2}$ is the convolution product in $S(G)$.

For $f \in S(G)$ we define $\tilde{f}(g)=f\left(i\left(g^{-1}\right)\right)$. Since the distribution $f \longmapsto m\left(F_{\ell}(f)\right)$ is $H$-bi-invariant, our hypotheses imply it is fixed by $i$. Thus $m\left(F_{\ell}(f)\right)=m\left(F_{\ell}(\tilde{f})\right)$. Now take $f=f_{1} * f_{2}$; since $i$ is an anti-involution we have $\tilde{f}=\tilde{f}_{2} * \tilde{f}_{1}$. Hence $B\left(f_{1}, f_{2}\right)=B\left(\tilde{f}_{2}, \tilde{f}_{1}\right)$; and in particular, the left kernel of $B$ (which is the kernel of $F_{m}$ ) determines the right kernel of $B$ (which is the kernel of $F_{\ell}$ ). Therefore $m$ determines the kernel of $F_{\ell}$, and hence $\ell$ up to scaling. Since $m$ was arbitrary, dim $\operatorname{Hom}_{H}(V, \mathbf{C})=1$. Similarly $\operatorname{dim} \operatorname{Hom}_{H}\left(V^{\prime}, \mathbf{C}\right)=1$.

When the subgroup $H$ of $G$ is compact, there are many simplifications. For example, one can show $i$ acts trivially on $H$-biinvariant distributions if and only if it acts trivially on $H \backslash G / H$. We also have the following.

Proposition 4.3. Assume that $(G, H)$ is a Gelfand pair, with $H$ compact. Let $V$ be an irreducible representation of $G$. Then

(a) $\operatorname{dim} \operatorname{Hom}_{H}(V, \mathbf{C})=\operatorname{dim} V^{H}$

(b) $\operatorname{dim} \operatorname{Hom}_{H}(V, \mathbf{C})=\operatorname{dim} \operatorname{Hom}_{H}\left(V^{\prime}, \mathbf{C}\right) \leq 1$.

(c) If $\ell: V \rightarrow \mathbf{C}$ is any $H$-invariant linear form, then $\ell$ lies in $V^{\prime}$.

Proof. (a) The projector $p(v)=\int_{H}(h v) d h$ from $V$ to $V^{H}$ allows one to identify $\operatorname{Hom}_{H}(V, \mathrm{C})$ with the dual of $V^{H}$. 
(b) Assume $\operatorname{Hom}_{H}(V, \mathbf{C}) \neq 0$ and let $v_{H}$ be a nonzero vector in $V^{H}$. Then $\ell_{H}^{\prime}=\left\langle v_{H}, \cdot\right\rangle: V^{\prime} \longrightarrow \mathbf{C}$ is nonzero and $H$ invariant, so $\operatorname{Hom}_{H}\left(V^{\prime}, \mathbf{C}\right) \neq 0$. Since $(G, H)$ is a Gelfand pair we must have $\operatorname{dim} \operatorname{Hom}_{H}(V, \mathbf{C})=\operatorname{dim} \operatorname{Hom}_{H}\left(V^{\prime}, \mathbf{C}\right)=1$. Reversing the roles of $V$ and $V^{\prime}$ shows that $\operatorname{Hom}_{H}(V, \mathbf{C})=0$ implies that $\operatorname{Hom}_{H}\left(V^{\prime}, \mathbf{C}\right)=0$, so the dimensions are always equal.

(c) Assume $\ell$ is nonzero. By part (b) the space $\left(V^{\prime}\right)^{H}$ has dimension 1. Let $v_{H}^{\prime}$ be a basis vector. Then $\ell$ is a multiple of the linear form $\left\langle, v_{H}^{\prime}\right\rangle: V \rightarrow \mathbf{C}$, so lies in $V^{\prime}$.

Proposition 4.4. If $H$ is compact and open in $G$, then $H$ is a Gelfand subgroup if and only if the Hecke algebra $L(H \backslash G / H)$ of locally constant, compactly supported, bi- $\mathrm{H}$-invariant functions on $G$ is commutative under convolution.

Proof. The functor $V \longmapsto V^{H}$ establishes an equivalence of categories between representations $V$ of $G$ which are generated by $V^{H}$ and representations of the algebra $L(H \backslash G / H)$ [C, 1.3]. This algebra is commutative if and only if its irreducible representations all have dimension 1 .

If $H$ is co-compact in $G$, we have the (smooth, admissible) representation $L(H \backslash G)$ of $G$ by right translation on the functions $f$ on $H \backslash G$ which are invariant by some open compact subgroup $K_{f}$. We have the following analog of Frobenius reciprocity

$$
\operatorname{Hom}_{G}(V, L(H \backslash G))=\operatorname{Hom}_{H}(V, \mathbf{C}) \text {. }
$$

In particular, the hypothesis that $L(H \backslash G)$ is multiplicity-free implies that $(G, H)$ is a Gelfand pair. This condition arises frequently in the theory of automorphic forms.

We should check that our definitions in this section are compatible with those in $\S 1$, in the case when $G$ is both compact and totally disconnected (i.e., $G$ is profinite). Then $H$ is necessarily both open and compact. Any irreducible representation $V$ of $G$ is finite dimensional; hence $V$ has a unique unitary structure and all linear maps from $V$ to a Hilbert space are continuous. Hence conditions (1.2), (1.3), (1.4), (4.1), (4.3), and (4.4) are all equivalent. Furthermore, Proposition 4.2 is equivalent to Lemma 2.1.

Since our notions are equivalent, we can consider locally compact groups $G$ of the type $G_{c} \times G_{d}$ where $G_{c}$ is compact and $G_{d}$ is totally disconnected. An irreducible representation of $G$ 
will be one of the form $V=V_{c} \otimes V_{d}$, where $V_{c}$ is a finite dimensional irreducible representation of $G_{c}$ and $V_{d}$ an irreducible smooth admissible representation of $G_{d}$. A closed subgroup $H$ of $G$ is Gelfand iff condition (4.1) holds for all irreducible $V$, with $V^{\prime}=V_{c}^{*} \otimes V_{d}^{\prime}=V_{c}^{\prime} \otimes V_{d}^{\prime}$.

Proposition 4.6. The group $G$ is a Gelfand subgroup of $G \times G$, under the diagonal embedding. The irreducible representations of $G \times G$ with a G-invariant linear form are those of the type $V \otimes V^{\prime}$. Proof. This is clear, as a $G$-invariant form on $V \otimes W$ gives an isomorphism of $V$ with $W^{\prime}$ (the unique smooth submodule in $\left.W^{*}\right)$. Note that, in this case, the contragredient of $V \otimes V^{\prime}$ is $V^{\prime} \otimes V$, and that any $G$-invariant form is a multiple of $\langle$,$\rangle .$

\section{EXAMPLES IN THE DISCONNECTED CASE}

In this section we give some examples which arise in number theory; this material will be pursued further in $\S \S 10-11$.

Let $F$ be a nonarchimedean local field, with ring of integers $A$ and finite residue field of characteristic $p$. If $F$ has characteristic zero, it is a finite extension of the field $\mathbf{Q}_{p}$ of $p$-adic numbers; if $F$ has characteristic $p$, it is a finite extension of the field $\mathbf{F}_{p}((t))$ of finite-tailed Laurent series over $\mathbf{F}_{p}$.

Let $\underline{G}$ be a connected, reductive algebraic group over $F$. Then the group $G=\underline{G}(F)$ of $F$-rational points is locally compact and totally disconnected [Sp, §4]. In fact, $G$ is compact if and only if $\underline{G}$ is $F$-anisotropic (i.e., $\underline{G}$ has no $F$-rational characters or cocharacters). We apply this to the algebraic group $\underline{G}=P G L_{2}$ and its tori below.

Proposition 5.1. Let $G=P G L_{2}(F)=G L_{2}(F) / F^{*}$.

(a) $H=P G L_{2}(A)$ is a compact open subgroup of $G$, and $(G, H)$ is a Gelfand pair. The Hecke algebra $L(H \backslash G / H)$ is isomorphic to the polynomial ring $\mathbf{C}[T]$.

(b) If $K$ is a separable quadratic $F$-algebra, then $H=K^{*} / F^{*}$ embeds as a closed subgroup of $G$, which is compact if and only if $K$ is a field. Furthermore, $(H \times G, H)$ is a Gelfand pair.

(c) $G$ is a Gelfand subgroup of $G \times G \times G$.

Proof. (a) The group $G$ acts 2-point transitively on the vertices of the tree associated to lattices in $F^{2}$ and $H=\operatorname{PGL}_{2}(A)$ is the stabilizer of the vertex corresponding to the lattice $A^{2}$ [S, Chapter II, §1]. Thus $(G, H)$ is Gelfand; the Hecke algebra is determined in [S, p. 73]. 
(b) This is proved using Proposition 4.2 and the anti-involution $i(h, g)=\left(h, \alpha g^{-1} \alpha^{-1}\right)$.

(c) This is proved using Proposition 4.2 and the anti-involution $i\left(g_{1}, g_{2}, g_{3}\right)=\left(g_{1}^{-1}, g_{2}^{-1}, g_{3}^{-1}\right)$. See [P, Chapter 4] for details.

Now assume that $E$ is a global field with no complex places. If $E$ has characteristic zero it is a finite, totally real, extension of $\mathbf{Q}$; if $E$ has characteristic $p$ it is a finite extension of the field $\mathbf{F}_{p}(t)$ of rational functions over $\mathbf{F}_{p}$. Let $\underline{G}$ be a connected, reductive algebraic group over $E$ and let $E_{\mathrm{A}}$ denote the ring of adèles of $E$. In the function field case, $G=\underline{G}\left(E_{\mathrm{A}}\right)$ is locally compact and totally disconnected [Sp, $\S 4]$. In the numberfield case, we assume that $\underline{G}(E \otimes \mathbf{R})$ is compact. Then $G=\underline{G}\left(E_{\mathrm{A}}\right)$ is the direct product of a compact group $G_{\infty}=\underline{G}(E \otimes \mathbf{R})$ with a totally disconnected group $G_{f}=\underline{G}(E \otimes \widehat{\mathbf{Z}})$. In particular, the results in $\S 4$ apply to the locally compact group $G$.

The group $\underline{G}(E)=I$ is a discrete subgroup of $G$, and $G / I$ is compact when $\underline{G}$ is anisotropic over $E$ [Sp, $\S 4]$.

Let $D$ be a quaternion division algebra over $E$ which is ramified at all real places (i.e., $D \otimes_{E} \mathbf{R}$ is a division algebra for all maps $E \rightarrow \mathbf{R})$. Let $\underline{G}$ be the algebraic group over $E$ with points $\underline{G}(F)=(D \otimes F)^{*} / F^{*}$ in any $E$-algebra $F$. If $D_{\mathrm{A}}=D \otimes E_{\mathrm{A}}$, then $G=G(\mathbf{A})=D_{\mathbf{A}}^{*} / E_{\mathbf{A}}^{*}$.

Proposition 5.2. Let $G=D_{\mathrm{A}}^{*} / E_{\mathrm{A}}^{*}$. (a) The subgroup $H=D^{*} / E^{*}$ is discrete and co-compact in $G$, and $(G, H)$ is a Gelfand pair.

(b) If $K$ is a separable quadratic field extension of $E$ which is contained in $D$, then $H=K_{\mathbf{A}}^{*} / E_{\mathbf{A}}^{*}$ is a compact subgroup of $G$ and $(H \times G, H)$ is a Gelfand pair.

(c) $G$ is a Gelfand subgroup of $G \times G \times G$.

Proof. (a) This is equivalent to the important theorem that any irreducible representation $V$ of $G$ occurs at most once in the space $L(H \backslash G)$ of automorphic forms (multiplicity one for inner forms of $\left.\mathrm{PGL}_{2}\right)[\mathrm{J}-\mathrm{L}]$.

(b) and (c) follow from the local theorems (Propositions 3.1 and 5.1 ), as $V$ decomposes uniquely as a restricted tensor product of representation of the local groups $D_{\wp}^{\times} / E_{\wp}^{\times}$.

\section{SPHERICAL FUNCTIONS AND DISTRIBUTIONS}

We assume throughout this section that $G$ is a locally compact group, which is the product of a compact group and a totally disconnected group, that $H$ is a Gelfand subgroup of $G$ in the sense 
of $\S \S 1$ and 4, and that $V$ is an irreducible representation of $G$ with $\operatorname{Hom}_{H}(V, \mathbf{C}) \neq 0$ and $\operatorname{Hom}_{H}\left(V^{\prime}, \mathbf{C}\right) \neq 0$. We let $\ell_{H}$ and $\ell_{H}^{\prime}$ be a basis of these one-dimensional spaces.

If $H$ is compact, by (4.3) there is a unique $H$-invariant vector $v_{H}$ in $V$ with $\ell_{H}\left(v_{H}\right)=1$. We define the spherical function $F$ of $V$ by the formula

$$
F(g)=\ell_{H}\left(g v_{H}\right)=g^{-1} \ell_{H}\left(v_{H}\right) .
$$

Clearly $F$ is bi- $H$-invariant and satisfies $F(e)=1$. If there is a $G$-invariant unitary structure (|) on $V$ (which is always the case when $G$ is compact), the linear form $\ell_{H}$ is given by $\ell_{H}(w)=$ $\left(w \mid v_{H}\right) /\left(v_{H} \mid v_{H}\right)$. Hence

$$
F(g)=\frac{\left(g v_{H} \mid v_{H}\right)}{\left(v_{H} \mid v_{H}\right)}
$$

when $V$ is unitary; in this case, the function $F$ also satisfies

$$
F\left(g^{-1}\right)=\overline{F(g)} \text { and }|F(g)| \leq 1 .
$$

When $H$ is noncompact, there is no spherical function $F$ associated to $V$, but we can define a spherical distribution $\Delta$ on $G$ which is $H$-bi-invariant. Let $f$ be an element in the space $S(G)$ of locally constant, compactly supported functions on $G$. The linear form

$$
v_{f}^{\prime}=\int_{G} f(g) g^{-1} \ell_{H} d g
$$

on $V$ then belongs to $V^{\prime}$. We define the spherical distribution $\Delta$ by

$$
\Delta(f)=\ell_{H}^{\prime}\left(v_{f}^{\prime}\right) .
$$

Then $\Delta$ is clearly $H$-bi-invariant. It depends, up to a nonzero scalar, on the choice of basis elements $\ell_{H}$ and $\ell_{H}^{\prime}$, as well as the choice of Haar measure on $G$ used to define the integral (6.4).

If $H$ is compact, we may take $\ell_{H}^{\prime}=\left\langle v_{H}, \cdot\right\rangle$. In this case we find

$$
\Delta(f)=\int_{G} f(g) F(g) d g
$$

where $F$ is the spherical function defined in (6.1). Even when $H$ is noncompact, the distribution $\Delta$ is frequently represented by a locally $L^{1}$-function $F$ on $G$ which is real analytic or locally constant outside of some specified singular set. For a discussion of 
how spherical functions may be used to recover the representation $V$, and to do "Fourier analysis" on the function space

$$
L^{1}(H \backslash G / H),
$$

see $[\mathrm{G}-\mathrm{V}]$.

\section{SOME CLASSICAL EXAMPLES OF SPHERICAL FUNCTIONS}

Let $(G, H)$ be a Gelfand pair. The determination of the irreducible representations $V$ of $G$ which have an $H$-invariant linear form, and the calculation of their spherical functions (or distributions) is a central problem in representation theory. One of the first examples studied was the compact group $\mathrm{G}=\mathrm{SO}_{3}$ and its Gelfand subgroup $\mathrm{H}=\mathrm{SO}_{2}$. In this case there is an irreducible representation $V_{\ell}$ of $G$ for every integer $\ell \geq 0$. This representation is self-dual, and is uniquely determined by its dimension, which is equal to $2 \ell+1$. We have $\operatorname{dim} V_{\ell}^{H}=1$ for all $\ell$. Finally, the spherical function $F_{\ell}$ of $V_{\ell}$ is given by the formula

$$
F_{\ell}(g)=P_{\ell}\left(\cos \varphi_{g}\right) \text {, }
$$

where $P_{\ell}(t)$ is the $\ell$ th Legendre polynomial and $\varphi_{g}$ is the Euler angle of the point $g(0,0,1)$ on the 2-sphere $X$ in $\mathbf{R}^{3}$. Proofs of all these claims can be found in [D, Chapter 2].

Next, consider the Gelfand pair $(G, H)=(H \times H, H)$. The irreducible representations of $G$ with an $H$-fixed linear form are precisely those of the form $V \otimes V^{\prime}$, where $V$ is an irreducible representation of $H$. In this case $\ell_{H}=\langle$,$\rangle is the canonical$ bilinear form on $V \times V^{\prime}$; the associated spherical distribution $\Delta$ on $G$ which is $H$-bi-invariant can be identified with a distribution $\chi$ on $H$ which is invariant under conjugation. If $H$ is compact, we may take

$$
v_{H}=\frac{1}{\operatorname{dim} V} \sum_{i=1}^{n} e_{i} \otimes e_{i}^{\vee},
$$

where $e_{i}$ is a basis for $V$ and $e_{i}^{\vee}$ is the dual basis of $V^{\prime}$. We then have the formula

$$
F(h, 1)=\frac{1}{\operatorname{dim} V} \sum_{i=1}^{n}\left\langle h e_{i}, e_{i}^{\vee}\right\rangle
$$

so

$$
\chi(h)=\frac{1}{\operatorname{dim} V} \operatorname{Trace}(h \mid V) .
$$


When $H$ is not compact there are generally no $H$-fixed vectors in $V \otimes V^{\prime}$, and the distribution $\chi$ is not represented by a spherical function on all of $H$. However, when $H$ is a semisimple $p$ adic Lie group, Harish-Chandra proved that the distribution $\chi$ is represented by a locally constant function on the set of regular elements in $H$.

\section{Correlation CONSTANTS}

In this section, we assume $G$ has two Gelfand subgroups $H$ and $I$, and $V$ is an irreducible representation of $G$ with $\operatorname{Hom}_{H}(V, \mathbf{C})$, $\operatorname{Hom}_{H}\left(V^{\prime}, \mathbf{C}\right), \operatorname{Hom}_{I}(V, \mathbf{C})$, and $\operatorname{Hom}_{I}\left(V^{\prime}, \mathbf{C}\right)$ all of dimension 1. We let $\ell_{H}, \ell_{H}^{\prime}, \ell_{I}$, and $\ell_{I}^{\prime}$ be bases of these vector spaces.

If $H$ and $I$ are both compact, then $\ell_{H}^{\prime}$ and $\ell_{I}^{\prime}$ correspond to vectors $v_{H}$ and $v_{I}$ in $V$ which are fixed by $H$ and $I$, respectively. We define the correlation constant

$$
c=c(V ; H, I)=\frac{\ell_{H}\left(v_{I}\right) \ell_{I}\left(v_{H}\right)}{\ell_{H}\left(v_{H}\right) \ell_{I}\left(v_{I}\right)} .
$$

If $V$ has a $G$-invariant unitary structure $(\mid)$, which is always true when $G$ is compact, we have the formula:

$$
c=\frac{\left(v_{I} \mid v_{H}\right)}{\left(v_{H} \mid v_{H}\right)} \cdot \frac{\left(v_{H} \mid v_{I}\right)}{\left(v_{I} \mid v_{I}\right)} .
$$

In this case $0 \leq c \leq 1$; if $V=V^{*}$ is defined over $\mathbf{R}, c$ is the square of the cosine of the angle $\theta$ between the lines fixed by $H$ and $I$ :

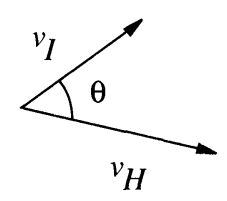

If we only assume that the subgroup $H$ is compact, we cannot define $c$ precisely. But since $\ell_{H} \in V^{\prime}$ corresponds to a fixed vector $v_{H}$ in $V$, one can ask if the value

$$
\ell_{I}^{\prime}\left(\ell_{H}\right) \cdot \ell_{I}\left(v_{H}\right)
$$

is nonzero in $\mathbf{C}$. If this is the case, we say that the subgroups $H$ and $I$ are correlated with respect to $V$; this is equivalent to the condition that $c \neq 0$ when both $H$ and $I$ are compact. 
In fact, we can test for correlation under the weaker hypothesis that

The quotient $H / H \cap I$ is compact and has an $H$ invariant measure.

In the case when (8.5) holds, we say the subgroups are correlated with respect to $V$ if and only if

The linear forms $A v_{H}\left(\ell_{I}\right)=\int_{H / H \cap I}\left(h \ell_{I}\right) d h$ and

$$
A v_{H}\left(\ell_{I}^{\prime}\right)=\int_{H / H \cap I}\left(h \ell_{I}^{\prime}\right) d h \text { are nonzero in }
$$

$\operatorname{Hom}_{H}(V, \mathbf{C})$ and $\operatorname{Hom}_{H}\left(V^{\prime}, \mathbf{C}\right)$, respectively.

Equivalently, we say $H$ and $I$ are correlated in $V$ if and only if

The linear form $A v_{H \times H}\left(\ell_{I} \otimes \ell_{I}^{\prime}\right)$ is nonzero in $\mathrm{Hom}_{H \times H}\left(V \otimes V^{\prime}, \mathbf{C}\right)$.

This is clearly equivalent to condition (8.4) when $H$ is compact, as we can test if a form is nonzero in $\operatorname{Hom}_{H \times H}\left(V \otimes V^{\prime}, \mathbf{C}\right)$ by evaluation on the vector $v_{H} \otimes \ell_{H}$.

The following example of correlation is quite useful. Let $I$ be a Gelfand subgroup of $G$ and let $V$ be an irreducible representation of $G$ with $\operatorname{Hom}_{I}(V, \mathbf{C})$ and $\operatorname{Hom}_{I}\left(V^{\prime}, \mathbf{C}\right)$ both of dimension one. We consider $I^{2}$ and $G$ as two Gelfand subgroups of $G^{2}$, and want to know if they are correlated with respect to the representation $V \otimes V^{\prime}$. When $G$ is compact, a simple calculation shows that

$$
c\left(V \otimes V^{\prime} ; G, I^{2}\right)=1 / \operatorname{dim} V .
$$

Similarly, these subgroups are correlated under the weaker hypothesis that $I$ is compact: indeed, $\ell_{G}\left(v_{I^{2}}\right)=\left\langle v_{I}, \ell_{I}\right\rangle \neq 0$. If $I$ is co-compact in $G$, they are correlated if and only if the linear form

$$
A v_{G}\left(\ell_{I} \otimes \ell_{I^{\prime}}\right)=\int_{G / I}\left(g \ell_{I} \otimes g \ell_{I}^{\prime}\right) d g
$$

is a nonzero multiple of $\langle$,$\rangle in \operatorname{Hom}_{G}\left(V \otimes V^{\prime}, \mathbf{C}\right)$. This is certainly the case when $V$ is a unitary representation of $G$.

\section{AN EXAMPLE. RACAH'S FORMULA FOR THE CORRELATION CONSTANT}

Let $G=S_{3}^{3}$ and consider the two Gelfand subgroups $H=$ $\mathrm{SO}_{2}^{3}$ and $\mathrm{I}=\mathrm{SO}_{3}$ in $\mathrm{G}$. The irreducible representations $\mathrm{V}$ of $G$ have the form $V_{\ell_{1}} \otimes V_{\ell_{2}} \otimes V_{\ell_{3}}$, where $V_{\ell}$ is the unique irreducible 
representation of $\mathrm{SO}_{3}$ of dimension $2 \ell+1$. Every $V$ has a unique line fixed by $H$; it has a line fixed by $I$ if and only if $\ell_{i} \leq \ell_{j}+\ell_{k}$ for all $i \neq j \neq k$. This follows from the self-duality of $V_{\ell_{3}}$ and the Clebsch-Gordan formula

$$
V_{\ell_{1}} \otimes V_{\ell_{2}}=V_{\ell_{1}+\ell_{2}} \oplus V_{\ell_{1}+\ell_{2}-1} \oplus \cdots \oplus V_{\left|\ell_{1}-\ell_{2}\right|} .
$$

We henceforth assume this is the case, so the integers $\left(\ell_{1}, \ell_{2}, \ell_{3}\right)$ form the sides of a (possibly degenerate) Euclidean triangle.

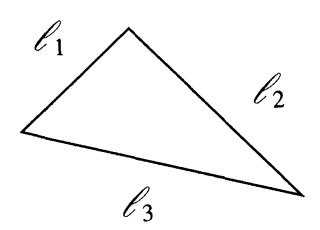

We now wish to calculate the correlation coefficient

$$
c=c(V ; H, I)
$$

defined in (8.1). A painful comparison with the physics literature [B-L, 3.12] shows that $c$ is given by the square of a Wigner $3-j$ symbol:

$$
c=\left(\begin{array}{ccc}
\ell_{1} & \ell_{2} & \ell_{3} \\
0 & 0 & 0
\end{array}\right)^{2}
$$

When $\ell_{1}+\ell_{2}+\ell_{3}$ is odd, it is not difficult to show $c=0$. When $\ell_{1}+\ell_{2}+\ell_{3}=2 n$ is even, we may solve the equations

$$
\left\{\begin{array}{l}
\ell_{1}=n_{2}+n_{3}, \\
\ell_{2}=n_{1}+n_{3}, \\
\ell_{3}=n_{1}+n_{2},
\end{array}\right.
$$

uniquely for integers $n_{i} \geq 0$ with $n_{1}+n_{2}+n_{3}=n$. Then Racah's formula [B-L, 3.194] for $c$ is given by

$$
c=\frac{\left(\begin{array}{c}
2 n_{1} \\
n_{1}
\end{array}\right)\left(\begin{array}{c}
2 n_{2} \\
n_{2}
\end{array}\right)\left(\begin{array}{c}
2 n_{3} \\
n_{3}
\end{array}\right)}{(2 n+1)\left(\begin{array}{c}
2 n \\
n
\end{array}\right)}
$$

In particular, $c$ is always a rational number.

One can obtain all the (squares of the) Wigner $3-j$ symbols as correlation constants for the subgroups $\mathrm{H}=\mathrm{SO}_{2}^{3}$ and $\mathrm{I}=$ $\mathrm{SO}_{2} \times \mathrm{SO}_{3}$ in $\mathrm{G}=\left(\mathrm{SO}_{2} \times \mathrm{SO}_{3}\right)^{3}$.

10. LOCAL PAIRS AND $\epsilon$-FACTORS

We now consider some of the arithmetic Gelfand pairs introduced in $\S 5$. Our first task is to determine which irreducible 
representations $V$ of $G$ have a nonzero $H$-invariant linear form. This will turn out to have a surprising connection with local $\epsilon$ factors.

Let $F$ be a nonarchimedean local field, with ring of integers A.

Proposition 10.1. Let $H=P G L_{2}(F)$ and let $G=H^{2}$. The irreducible representation $V=V_{1} \otimes V_{2}$ of $G$ has a nonzero $H$-invariant linear form, which is unique up to scaling, if and only if $V_{2} \simeq V_{1}^{\prime}$. Proof. This is true for any pair $H \hookrightarrow H \times H$. We note that all representations of $\mathrm{PGL}_{2}(F)$ are isomorphic to their contragredients, so we have

$$
\operatorname{Hom}_{H}\left(V_{1} \otimes V_{2}, \mathbf{C}\right) \neq 0 \quad \text { iff } V_{1} \simeq V_{2} .
$$

We now consider irreducible representations $V=V_{1} \otimes V_{2} \otimes V_{3}$ of the group $G=H^{3}=\mathrm{PGL}_{2}(F)^{3}$. We will assume that all $V_{i}$ are infinite dimensional; otherwise $\operatorname{dim} V_{i}=1$ and we are reduced to (10.1). Associated to any such representation, we have its Langlands' parameter $\varphi=\left(\varphi_{1}, \varphi_{2}, \varphi_{3}\right)$, which is a homomorphism from the Weil-Deligne group $W_{F}^{\prime}$ of $F$ to the $L$-group ${ }^{L} G(C)=\mathrm{SL}_{2}(C){ }^{3}$. The triple tensor product of the three standard representations of $\mathrm{SL}_{2}(C)$ gives an 8-dimensional symplectic representation $r$ of ${ }^{L} G(\mathbf{C})$, and hence the composition $\sigma=$ $r \circ \varphi: W_{F}^{\prime} \longrightarrow \mathrm{Sp}_{8}(\mathbf{C})$ gives a symplectic representation of the Weil-Deligne group associated to $V$. We define the local $\epsilon$-factor [Ta]:

$$
\epsilon(V)=\epsilon(\sigma, \psi, d x),
$$

where $\psi$ is a nontrivial additive character of $F$ and $d x$ is the unique Haar measure on $F$ which is self-dual with respect to Fourier transform using the duality $(x \mid y)=\psi(x y)$. Then $\epsilon(V)$ is independent of the choice of $\psi$ and satisfies $\epsilon(V)^{2}=1$.

Proposition 10.4 (Prasad [P]). Let $H=P G L_{2}(F)$ and let $G=H^{3}$. The irreducible representation $V=V_{1} \otimes V_{2} \otimes V_{3}$ of $G$ is self-dual $\left(V \simeq V^{\prime}\right)$. It has a nonzero $H$-invariant linear form, which is unique up to scaling, if and only if $\epsilon(V)=+1$.

When $\epsilon(V)=-1$, the Langlands parameters $\varphi_{i}$ cannot be reducible and hence each representation $V_{i}$ lies in the discrete series for $\mathrm{PGL}_{2}(F)$. By a theorem of Jacquet and Langlands, $V_{i}$ corresponds to a unique irreducible representation $W_{i}$ of the compact 
form $D^{*} / F^{*}$ of $\mathrm{PGL}_{2}(F)$ (where $D$ is the unique quaternion division algebra with center $F$ ). More generally, given an irreducible representation $W=W_{1} \otimes W_{2} \otimes W_{3}$ of $\left(D^{*} / F^{*}\right)^{3}$, we define $\epsilon(W)=\epsilon(V)= \pm 1$ where $V=V_{1} \otimes V_{2} \otimes V_{3}$ is the corresponding product of discrete series representations for $\mathrm{PGL}_{2}(F)$.

Proposition 10.5 (Prasad). Let $F$ be a local field and $D$ a quaternion division algebra over $F$. Let $H=D^{*} / F^{*}$ and let $G=H^{3}$. The irreducible representation $W=W_{1} \otimes W_{2} \otimes W_{3}$ of $G$ is self-dual. It has a nonzero $H$-invariant linear form, which is unique up to scaling, if and only if $\epsilon(W)=-1$.

There are similar results for the Gelfand pair $(H \times G, H)$ where $H=K^{*} / F^{*}$ is the torus in $G=\mathrm{PGL}_{2}(F)$ defined by a separable quadratic extension $K$. Associated to an irreducible representation $V=\chi_{1} \otimes V_{2}$ of $H \times G$ with $V_{2}$ infinite dimensional, we have the Langlands parameter $\varphi=\left(\varphi_{1}, \varphi_{2}\right)$, where $\varphi_{1}=\chi_{1}$ is a homomorphism from $W_{K}^{\prime}$ to $\mathrm{GL}_{1}(\mathbf{C})$ and $\varphi_{2}$ is a homomorphism from $W_{F}^{\prime}$ to $\mathrm{SL}_{2}(\mathrm{C})$. The tensor product of $\varphi_{2}$ with the representation induced from $\varphi_{1}$ gives a 4-dimensional symplectic representation $\sigma=\operatorname{Ind}_{K}^{F} \varphi_{1} \otimes \varphi_{2}: W_{F}^{\prime} \longrightarrow \operatorname{Sp}_{4}(C)$ associated to $V$. Indeed, Ind $\varphi_{1}$ is an orthogonal representation, with determinant equal to the quadratic character $\omega_{K / F}$ associated to the extension $K$. We define

$$
\epsilon(V)=\epsilon(\sigma, \psi, d x)
$$

as above; again, this depends only on $V$ and satisfies $\epsilon(V)^{2}=1$. The dual representation $V^{\prime}$ of $V$ is equal to $\chi_{1}^{-1} \otimes V_{2}$; since $\operatorname{Ind}_{K}^{F} \varphi_{1}=\operatorname{Ind}_{K}^{F} \varphi_{1}^{-1}$ we have $\epsilon\left(V^{\prime}\right)=\epsilon(V)$.

Proposition 10.7 (Waldspurger [W], Tunnell [T]). Let $H=K^{*} / F^{*}$ and $G=P G L_{2}(F)$. The irreducible representation $V=\chi_{1} \otimes V_{2}$ of $H \times G$ has a nonzero $H$-invariant linear form, which is unique up to scaling, if and only if $\epsilon(V)=\omega_{K / F}(-1)$.

If $\epsilon(V)=-\omega_{K / F}(-1)$ the representation $V_{2}$ is in the discrete series and the algebra $K$ is a field. The irreducible representation $W=\chi_{1} \otimes W_{2}$ of $H \times\left(D^{*} / F^{*}\right)$ has a nonzero $H$-invariant linear form, which is unique up to scaling.

Actually, the full statement in Proposition 10.7 has not been verified in the case when the residual characteristic of $F$ is 2 and when $V_{2}$ is a super-cuspidal representation. This would be a good exercise on exotic representations. 
We now give some results on adèlic groups, which are fairly simple corollaries of the above local propositions. Let $E$ be a global field with no complex places, and let $D$ be a quaternion division algebra over $E$ which is ramified at every archimedean place. For each place $\wp$ of $E$ we define $\delta\left(D_{\wp}\right)=1$ if $D_{\wp}=D \otimes E_{\wp}$ is a $2 \times 2$ matrix algebra over $E_{\wp}$ and $\delta\left(D_{\wp}\right)=-1$ if $D_{\wp}$ is a division algebra over $E_{\wp}$.

We recall the group $D_{\mathrm{A}}^{*} / E_{\mathrm{A}}^{*}$ defined in $\S 5$. An irreducible representation $V$ of this adèlic group decomposes uniquely as a restricted tensor product over the places $\wp$ of $E$ :

$$
V \simeq \widehat{\otimes} V_{\wp}
$$

where $V_{\wp}$ is an irreducible representation of the local group $D_{\wp}^{*} / E_{\wp}^{*}$ (which, by our hypotheses, is either compact or totally disconnected). Any collection of local representations $V_{\wp}$ can be combined to give a global representation $V$, provided almost all of the $V_{\wp}$ are unramified ( $=$ have a vector fixed by $\operatorname{PGL}_{2}\left(A_{\wp}\right)$ ). We assume that each $V_{\wp}$ is infinite dimensional.

Proposition 10.9. Let $H=D_{\mathrm{A}}^{*} / E_{\mathrm{A}}^{*}$ and let $G=H^{3}$. The irreducible representation $V=V_{1} \otimes V_{2} \otimes V_{3}$ of $G$ is self-dual. It has a nonzero $H$-invariant linear form, which is unique up to scaling, if and only if for all places $\wp$ of $E$ we have $\epsilon\left(V_{\wp}\right)=\delta\left(D_{\wp}\right)$.

In other words, a global linear form exists if and only if $H_{\wp}$ invariant forms exist on $V_{\wp}$ for all completions $\wp$. One proves this by taking the restricted product of local forms, using the result that, in the unramified case, $\ell_{H}$ is nontrivial on the vector fixed by $\mathrm{PGL}_{2}\left(A_{\wp}\right)^{3}$ [P, Gr-P].

Note 10.10. There are always an even number of places $\wp$ which are ramified in $D$ (i.e., where $\delta\left(D_{\wp}\right)=-1$ ). Hence a necessary condition on the existence of a global $H$-invariant linear form is that

$$
\epsilon(V)=\prod_{\wp} \epsilon\left(V_{\wp}\right)=+1 .
$$

The analogous result for tori is the following. Let $K$ be a separable quadratic field extension of $E$ which embeds as a subfield of $D$.

Proposition 10.12. Let $H=K_{\mathbf{A}}^{*} / E_{\mathbf{A}}^{*}$ and $G=D_{\mathbf{A}}^{*} / E_{\mathbf{A}}^{*}$. The irreducible representation $V=\chi_{1} \otimes V_{2}$ of $H \times G$ has a nonzero 
$H$-invariant linear form, which is unique up to scaling, if and only if for all places $\wp$ of $E$ we have $\epsilon_{\wp}(V)=\omega_{K_{\wp} / E_{\wp}}(-1) \cdot \delta_{\wp}(D)$.

Again we assume that all local components of $V_{2}$ are infinite dimensional. A necessary condition for the existence of an $\mathrm{H}$ invariant linear form is that

$$
\epsilon(V)=\prod_{\wp} \epsilon_{\wp}(V)=+1 \quad \text { as } \prod_{\wp} \omega_{\wp}(-1)=1
$$

by global classfield theory.

\section{AUTOMORPHIC CORRELATION}

Recall the adelic group $D_{\mathbf{A}}^{*} / E_{\mathbf{A}}^{*}$ defined in the last section, where $E$ is a global field and $D$ a quaternion division algebra over $E$ which is ramified at each Archimedean place. An irreducible representation $V$ of this group is called automorphic if there is a nonzero, $D^{*} / E^{*}$-invariant linear form $\ell: V \rightarrow \mathrm{C}$. Since we have seen that $\left(D_{\mathrm{A}}^{*} / E_{\mathrm{A}}^{*}, D^{*} / E^{*}\right)$ is a Gelfand pair, $\ell$ is unique up to scaling. Giving $\ell$ is equivalent, by Frobenius reciprocity (4.5), to giving an injection of $G$-modules from $V$ into the space of automorphic forms $L\left(D_{\mathrm{A}}^{*} / E_{\mathrm{A}}^{*} D^{*}\right)$. Indeed, we map $v \in V$ to the automorphic form $f_{v}(g)=g \ell(v)=\ell\left(g^{-1} v\right)$ [J-L].

The irreducible representations of the adelic groups $G$ studied in (10.9) and (10.12) have invariant linear forms for the adèlic subgroup $H$ if and only if invariant linear forms exist locally for $H_{\wp}$, for all places $\wp$ of $E$. To obtain global results, we will consider the correlation of the adelic subgroup $H$ with a discrete subgroup $I$ in an automorphic representation $V$. This gives a candidate $A v_{H}\left(\ell_{I}\right)$ for a nontrivial $H$-invariant form, and the nonvanishing of this average often has a surprising connection to the behavior of the $L$-function of $V$ in the center of its critical strip.

First, let $G=\left(D_{\mathrm{A}}^{*} / E_{\mathrm{A}}^{*}\right)^{2}$, and consider the two Gelfand subgroups $H=D_{\mathrm{A}}^{*} / E_{\mathrm{A}}^{*}$ (embedded diagonally) and $I=\left(D^{*} / E^{*}\right)^{2}$. The latter subgroup is discrete and co-compact in $G$. The irreducible representations $V$ of $G$ with $H$ and $I$-invariant linear forms are precisely those of the form $W \otimes W^{\prime}$, where $W$ is an automorphic representation of $D_{\mathbf{A}}^{*} / E_{\mathbf{A}}^{*}$.

Proposition 11.1. We have $A v_{H}\left(\ell_{I}\right) \neq 0$ in $\operatorname{Hom}_{H}(V, \mathbf{C})$. 
Proof. Since $W \simeq W^{\prime}$ is self-dual, we may define $V$ over $\mathbf{R}$. We have the formula

$$
A v_{H}\left(\ell_{I}\right)(v \otimes w)=\int_{D_{\mathbf{A}}^{*} / E_{\mathbf{A}}^{*} \cdot D^{*}} f_{v}(h) f_{w}(h) d h .
$$

The integral on the right is actually a finite sum, as $f_{v}$ and $f_{w}$ are invariant under an open compact subgroup of $D_{\mathrm{A}}^{*}$. Taking $v=w$ and using the fact that $f_{v}(h)^{2} \geq 0$, we see that $A v_{H}\left(\ell_{I}\right)(v \otimes v) \geq 0$ with equality for $v=0$. In particular, $A v_{H}\left(\ell_{I}\right) \neq 0$.

Next, consider the case when $G=\left(D_{\mathrm{A}}^{*} / E_{\mathbf{A}}^{*}\right)^{3}, H=D_{\mathrm{A}}^{*} / E_{\mathbf{A}}^{*}$ and $I=\left(D^{*} / E^{*}\right)^{3}$. The irreducible representations $V$ of $G$ with $H$ - and $I$-invariant linear forms are those of the type $V_{1} \otimes V_{2} \otimes$ $V_{3}$, with all $V_{i}$ automorphic and satisfying the local conditions of Proposition 10.9. Again $H \cap I=D^{*} / E^{*}$ is co-compact in $H$, so we may consider the form $A v_{H}\left(\ell_{I}\right)$ defined by (8.6).

Recall the 8-dimensional symplectic representation $r$ of the $L$ group $\mathrm{SL}_{2}(\mathrm{C})^{3}$ discussed preceding (10.3). Associated to $V$ and $r$, we may define the Langlands $L$-function

$$
L(V, s)=\prod_{\wp} L_{\wp}(\varphi, r, s)
$$

by an Euler product which converges for $\operatorname{Re}(s)$ large. Using a method initiated by Garrett [G], Piatetski-Shapiro and Rallis [PS$\mathrm{R}]$ have shown that $L(V, s)$ has a meromorphic continuation to the entire $s$-plane, with only a finite number of poles. This function satisfies a functional equation (as yet very imprecisely stated, but see [Gr-K] for a special case) when $s$ is replaced by $1-s$, and is regular at $s=\frac{1}{2}$.

Proposition 11.3 (Harris-Kudla $[\mathrm{H}-\mathrm{K}]$ ). We have $A v_{H}\left(\ell_{I}\right) \neq 0$ in $\operatorname{Hom}_{H}(V, \mathbf{C})$ if and only if the central critical value $L\left(V, \frac{1}{2}\right)$ is nonzero.

This beautiful result was conjectured by Jacquet. To translate into the language of his conjecture and the proof, we note that

$$
A v_{H}\left(\ell_{I}\right)\left(v_{1} \otimes v_{2} \otimes v_{3}\right)=\int_{D_{\mathbf{A}}^{*} / E_{\mathbf{A}}^{*} D^{*}} f_{1}(h) f_{2}(h) f_{3}(h) d h .
$$

Finally we consider the tori in $D_{\mathrm{A}}^{*}$. Let $K$ be a separable quadratic field extension of $E$ which embeds as a subfield of $D$. Let $G=K_{\mathrm{A}}^{*} / E_{\mathrm{A}}^{*} \times D_{\mathrm{A}}^{*} / E_{\mathrm{A}}^{*}$, and consider the two Gelfand subgroups $H=K_{\mathrm{A}}^{*} / E_{\mathrm{A}}^{*}$ (embedded diagonally) and $I=K^{*} / E^{*} \times D^{*} / E^{*}$. The irreducible representations $V$ of $G$ with $H$-and $I$-invariant 
linear forms are those of the type $\chi \otimes W$, where $\chi$ is a Hecke character of $K, W$ is an automorphic representation of $D_{\mathrm{A}}^{*}$, and $V$ satisfies the local conditions of Proposition 10.12. The contragredient $V^{\prime}$ of $V$ is given by $\chi^{-1} \otimes W$, and we may consider the linear forms $A v_{H}\left(\ell_{I}\right)$ and $A v_{H}\left(\ell_{I}^{\prime}\right)$ defined in (8.6).

Recall the 4-dimensional symplectic representation $r$ of the $L$ group $\mathrm{O}_{2}(\mathbf{C}) \times \mathrm{SL}_{2}(\mathbf{C})$ defined preceding (10.6). Associated to $V$ and $r$ we may define the Langlands $L$-function $L(V, s)=$ $\prod_{\wp} L_{\wp}(\varphi, r, s)=L\left(V^{\prime}, s\right)$ by a convergent Euler product. By Rankin's method, as extended by Jacquet [J], this function has a meromorphic continuation to the $s$-plane, satisfies a precise functional equation when $s$ is replaced by $1-s$, and is regular at $s=\frac{1}{2}$.

Proposition 11.5 (Waldspurger [W]). We have $A v_{H}\left(\ell_{I}\right)$ and $A v_{H}\left(\ell_{I}^{\prime}\right)$ nonzero if and only if the central critical value $L\left(V, \frac{1}{2}\right)=L\left(V^{\prime}, \frac{1}{2}\right)$ is nonzero.

To translate to the language of the proof, we note that

$$
A v_{H}\left(\ell_{I}\right)(1 \otimes v)=\int_{K_{\mathbf{A}}^{*} / E_{\mathbf{A}}^{*} K^{*}} \chi(h) f_{v}(h) d h
$$

with a similar formula (with $\chi$ replaced by $\chi^{-1}$ ) holding for $A v_{H}\left(\ell_{I}^{\prime}\right)(1 \otimes v)$.

In certain of the above cases, one can be much more precise. For example, assume the representation $V$ of $G$ considered has a vector fixed by a maximal compact open subgroup $K$. Then $\operatorname{dim} V^{K}=\operatorname{dim} V^{\prime} K=1$; let $v_{K}$ and $v_{K}^{\prime}$ be bases of this space. One can show easily that the linear form $A v_{G}\left(\ell_{I} \otimes \ell_{I}^{\prime}\right)$ is always nonzero on $v_{K} \otimes v_{K}^{\prime}$, and that the linear form $A v_{H}\left(\ell_{I}\right) \otimes A v_{H}\left(\ell_{I}^{\prime}\right)$ is nonzero if and only if it takes a nonzero value on $v_{K} \otimes v_{K}^{\prime}$ [Gr-P]. Thus the ratio

$$
c=c(V)=c\left(V^{\prime}\right)=\frac{A v_{H \times H}\left(\ell_{I} \otimes \ell_{I^{\prime}}\right)\left(v_{K} \otimes v_{K}^{\prime}\right)}{A v_{G}\left(\ell_{I} \otimes \ell_{I^{\prime}}\right)\left(v_{K} \otimes v_{K}^{\prime}\right)}
$$

is well defined, and is zero if and only if $L\left(V, \frac{1}{2}\right)=0$. Note that $c$ depends only on $V$.

As a specific example, assume $E=\mathbf{Q}$ and $D$ is the quaternion division algebra ramified at $\{\infty, p\}$. Let $V=V_{1} \otimes V_{2} \otimes V_{3}$ be an automorphic representation of $\left(D_{\mathrm{A}}^{*} / E_{\mathrm{A}}^{*}\right)^{3}$ which is unramified locally for all primes $\ell \neq p, \infty$, and is one-dimensional locally 
at both $\infty$ and $p$. Then each representation $V_{i}$ corresponds to a real-valued function $\lambda_{i}(e)$ on the set of supersingular elliptic curves $e(\bmod p)$, which is well defined up to a scaling [M-O]. Let $w(e)=\#(\operatorname{Aut}(e) / \pm 1)$; then

$$
c(V)=\frac{\left(\sum w(e)^{2} \lambda_{1}(e) \lambda_{2}(e) \lambda_{3}(e)\right)^{2}}{\sum w(e) \lambda_{1}(e)^{2} \sum w(e) \lambda_{2}(e)^{2} \sum w(e) \lambda_{3}(e)^{2}}
$$

where the sums are taken over the finite set of supersingular elliptic curves $e(\bmod p)$. For the specific relation between $c(V)$ and $L\left(V, \frac{1}{2}\right)$, see $[\mathrm{Gr}-\mathrm{K}]$.

Finally, we note that the notion of automorphic correlation has been studied in a more general setting by Jacquet [J2-J5]. Let $\underline{G}$ be a reductive group defined over a global field $E$, and let $\underline{H}$ be a reductive subgroup such that $(\underline{G}, \underline{H})$ forms an algebraic Gelfand pair. Put $H=\underline{H}\left(\mathbf{A}_{E}\right), G=\underline{G}\left(\mathbf{A}_{E}\right)$, and $I=\underline{G}(E)$. Jacquet calls an irreducible automorphic representation $V$ of $\underline{G}$ "distinguished" if there is a vector $f \in V \subseteq L(G / I)$ such that $\int_{\underline{H}\left(E_{\mathbf{A}}\right) / \underline{H}(E)} f(h) d h$ is nonzero. When $I$ and $H$ are both Gelfand subgroups of $\underline{G}$ and $H / H \cap I$ is compact, this is equivalent to the condition that $A v_{H}\left(\ell_{I}\right) \neq 0$. In general, one must distinguish between $V$ and its isomorphism class, and there may be convergence questions involved in evaluation of the integral. A natural method which Jacquet has introduced to study the distinguished components in the residual spectrum is the relative trace formula [J5]; he finds that the distinction of automorphic representations is often related to questions of Langlands' functorality.

\section{BIBLIOGRAPHY}

[B] J. N. Bernstein, P-invariant distributions on $G L_{n}$ and the classification of unitary representations of $G L_{n}$, Lecture Notes in Math., vol. 1041, Springer-Verlag, Berlin and New York, 1983.

[B-L] L. C. Biedenharn and J-D. Louck, Angular momentum in quantum physics, Encyclopedia Math. Appl., vol. 8, 1981.

[Bo] N. Bourbaki, Groupes et algèbres de Lie, Chapitres VII-VIII, Hermann, Paris, 1975.

[C] P. Cartier, Representations of $\wp$-adic groups, Proc. Sympos. Pure Math., vol. 33, Amer. Math. Soc., Providence, RI, 1979, pp. 111-155.

[Di] P. Diaconis, Group representations in probability and statistics, Lecture Notes 11, Institute of Mathematical Statistics, Hayward, CA, 1988.

[D] J. Dieudonné, Special functions and linear representations of Lie groups, CBMS Regional Conf. Ser., vol. 42, 1980. 
[G] P. Garrett, Decomposition of Eisenstein series: Rankin triple products, Ann. of Math. (2) 125 (1987), 209-235.

[G-V] R. Gangolli and V. S. Varadarajan, Harmonic analysis of spherical functions on real reductive groups, Ergeb. Math. Grenzgeb. vol. 101, Springer-Verlag, Berlin and New York, 1980.

[Ge] I. M. Gelfand, Spherical functions on symmetric spaces, Dokl. Akad. Nauk SSSR 70 (1950), 5-8.

[Ge-K] I. M. Gelfand and D. A. Kazhdan, Representations of $G L_{n}(K)$, Lie Groups and Their Representations, Halstead Press, 1975.

[Gr-K] B. H. Gross and S. Kudla, Heights and the central critical values of triple product L-functions, preprint, 1990.

[Gr-P] B. H. Gross and D. Prasad, Test vectors for linear forms, Preprint, 1990.

[H] S. Helgason, Differential geometry, Lie groups, and symmetric spaces, Academic Press, New York, 1978.

[H-K] M. Harris and S. Kudla, The central critical value of a triple product $L$ functions, preprint, 1989.

[J1] H. Jacquet, Automorphic forms on $G L_{2}$, Lecture Notes in Math., vol. 278, Springer-Verlag, Berlin and New York, 1972.

[J2] _ Sur un résultat de Waldspurger, Ann. Sci. École Norm. Sup. (Pisa) 19 (1986), 185-229.

[J3] _- Sur un résultat de Waldspurger. II, Compositio Math. 63 (1987), 315-389.

[J4] $\longrightarrow$ On the nonvanishing of some L-functions, Proc. Indian Acad. Sci. 97 (1987), 117-155.

[J5] H. Jacquet and K. F. Lai, A relative trace formula, Compositio. Math. 54 (1985), 243-310.

[J-L] H. Jacquet and R. P. Langlands, Automorphic forms on $G L_{2}$, Lecture Notes in Math., vol. 114, Springer-Verlag, Berlin and New York, 1970.

[K-V] B. Kimelfield and E. Vinberg, Homogeneous domains in flag manifolds and spherical subgroups of semisimple Lie groups, J. Funct. Anal. Appl. 12 (1978), 168-174.

[K] M. Krämer, Sphärische Untergruppen in Kompakten zusammenhängenden Lie Gruppen, Compositio. Math. 38 (1979), 129-153.

[L] S. Lang, $\mathrm{SL}_{2}(\mathbf{R})$, Addison-Wesley, Reading, MA, 1975.

[M] G. Mackey, Multiplicity free representations of finite groups, Pacific J. Math. 8 (1958), 503-510.

[M-O] J-F. Mestre and J. Oesterlé, Elliptic curves of prime conductor (in preparation).

[PS-R] I. Piatetski-Shapiro and S. Rallis, Rankin triple L-functions, Compositio Math. 64 (1987), 31-115.

[P] D. Prasad, Trilinear forms for $\mathrm{GL}_{2}$ of a local field and $\epsilon$-factors, Compositio Math., vol. 75, no. 1, 1990, pp. 1-46.

[S] J.-P. Serre, Trees, Springer-Verlag, Berlin and New York, 1980.

[Sp] T. A. Springer, Reductive groups, Automorphic Forms, Representations, and $L$-functions, Proc. Sympos. Pure Math., vol. 33, Amer. Math. Soc., Providence, RI, 1979, pp. 3-27.

[Ta] J. T. Tate, Number theoretic background, Proc. Sympos. Pure Math., vol. 33, Amer. Math. Soc., Providence, RI, 1979, pp. 3-26. 
[T] J. Tunnell, Local $\epsilon$-factors and characters of $G L_{2}$, Amer. J. Math. 105 (1983), 1277-1308.

[V] M.-F. Vigneras, Arithmétique des algèbres de quaternions, Lecture Notes in Math., vol. 800, Springer-Verlag, Berlin and New York, 1980.

[W] J.-L. Waldspurger, Sur les valeurs de certaines fonctions $L$ automorphes en leur centre de symétrie, Compositio Math. 54 (1985), 173-242.

Department of Mathematics, Harvard University, Cambridge, MasSACHUSETTS 02138

E-mail address: gross@zariski.harvard.edu 
